\title{
SIMULATION FOR AN RF GUN TEST APPARATUS IN THE SPring-8 LINAC
}

\author{
H. Abe, T. Asaka, H. Hanaki, A. Mizuno *, S. Suzuki, \\ T. Taniuchi, K. Yanagida, SPring-8, Hyogo, 679-5198, JAPAN
}

\section{Abstract}

A photo cathode rf gun has been studied in the SPring-8 Linac to obtain lower emittance beams. In order to compare an observed beam emittance or beam size by simulation, we are also developing a beam tracking code for this test apparatus. In this paper, we describe the outlines of our code, and some calculation results for our test apparatus.

\section{INTRODUCTION}

In the SPring-8 Linac, we are developing a photo cathode rf gun for a conventional injector for the linac, and also for future applications such as a single pass FEL based on the SASE. We simultaneously require lower emittance of around several $\pi \mathrm{mm} \cdot \mathrm{mrad}$ and a higher peak charge of around several nano Coulomb per bunch, especially for the SASE. In order to achieve these conditions, we must develop some calculation codes that can predict beam characteristics as accurately as possible. However, the readymade tracking codes are not considered sufficient, because a lot of assumptions are included. Thus, we have developed our own simulation code that is suitable for our rf gun apparatus and includes as few assumptions as possible.

\section{TEST APPARATUS}

The outline of our apparatus and an rf cavity [1] are shown in Fig.1. The s-band rf cavity is a single-cell cavity since the field distributions in the cavity are simpler than that for a multi cell-cavity, and this makes a comparison with simulations easier. The bottom side of the cavity, which is made of copper, is used as a cathode. The rf from the right hand side port travels to the opposite port and is fed to a dummy load.

There are two sets of $\mathrm{x}-\mathrm{y}$ slits for emittance measurement and a bending magnet for energy analyses.

\section{OUTLINE OF OUR CODE}

Our code is a three-dimensional particle tracking code that includes the production of electrons and space charge effects. As a result of recent increases in CPU speed, we try to calculate all space charge effects of each electron in the tracking code, but we assume that the charge and mass of each particle are larger than those of real electrons. The electron is accelerated in the cavity, but a calculation procedure for the space charge that includes acceleration becomes to be complicated so much. Thus, if we assume uni-

\footnotetext{
*Email: mizuno@spring8.or.jp
}

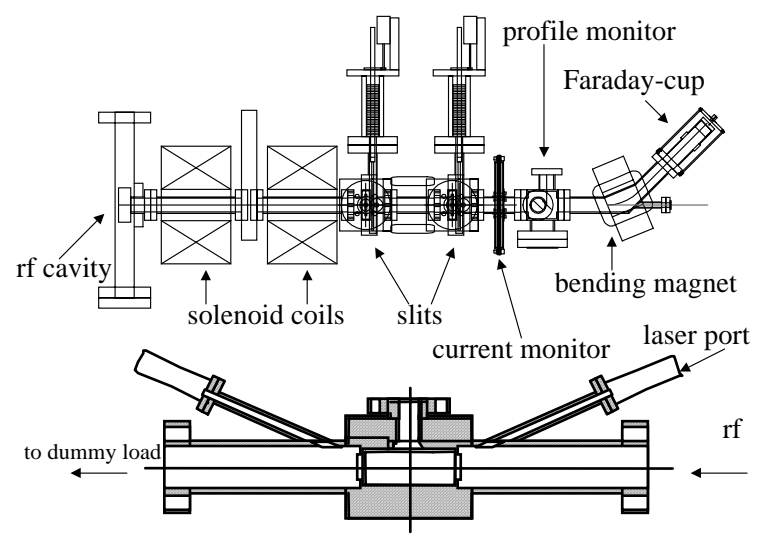

Figure 1: Rf gun test apparatus and cross section of rf cavity with two windows for laser injection.

form motion for electron, the electric and magnetic fields at point $A$ caused by electron $B$ are expressed as follows;

$\mathbf{E}_{A}=\frac{1}{4 \pi \epsilon_{0} \gamma^{2}} \frac{-e \mathbf{r}}{\left[|\mathbf{r}|^{2}-\frac{\left|\mathbf{v}_{B} \times \mathbf{r}\right|^{2}}{c^{2}}\right]^{3 / 2}} \quad \mathbf{B}_{A}=\frac{1}{c^{2}} \mathbf{v}_{B} \times \mathbf{E}_{A}$

where $\mathbf{r}$ is a vector from $B$ to $A, \mathbf{v}_{B}$ is a velocity of electron $B$, and $\gamma$ is a relative factor of electron $B$. These fields act on electron $A$ as follows:

$$
\mathbf{F}_{A}=-e\left(\mathbf{E}_{A}+\mathbf{v}_{A} \times \mathbf{B}_{A}\right)
$$

The equation of motion for each electron is derived to the following equation and becomes adaptable for the RungeKutta method.

$$
\begin{gathered}
-e(\mathbf{v} \times \mathbf{B}+\mathbf{E})=m_{0} \frac{d(\gamma \mathbf{v})}{d t} \\
\Downarrow \\
\frac{d \mathbf{v}}{d t}=-\frac{e}{\gamma m_{0}}\left(\mathbf{v} \times \mathbf{B}+\mathbf{E}-\frac{(\mathbf{v} \cdot \mathbf{E})}{c^{2}} \mathbf{v}\right)
\end{gathered}
$$

We consider two types of extra electromagnetic sources in our code. One is an rf cavity for a part of the electron production, and the other is a set of two coils for focusing the electron beam. The fields in the cavity are calculated in the MAFIA code and are included in our code. The fields of the solenoid coils are calculated in our code. We treat the extra fields in the same way for the fields due to space charge as shown above in Eq.3.

We also consider the image charge effect caused from the copper cathode in our code. 


\section{COMPARISON WITH OTHER CODES}

It is important to confirm the accuracy of our code by comparing it with other conventional codes. Fig.2 shows some comparisons with MAFIA's calculations, which include the electron production and tracking in the cavity. Table 1 lists parameters in their calculations.

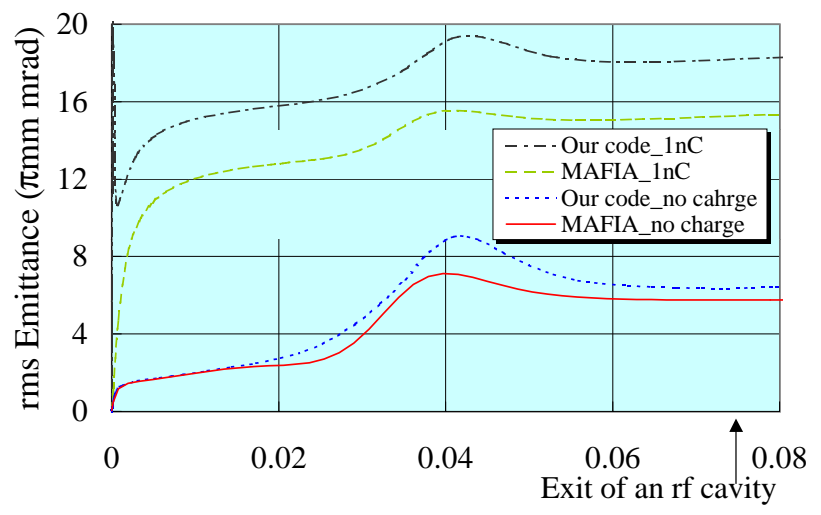

Distance from a cathode (m)

Figure 2: Comparison between our code and MAFIA ver4.0. An exit flange of rf cavity corresponds to 0.074 $\mathrm{m}$ in horizontal axis.

An initial rf phase $\phi$ in Table 1 is defined by

$$
E_{\text {cavity }}=E_{\text {max }} \cos (\omega t-\phi)
$$

To reduce memory size, MAFIA calculates in a twodimensional space, so the definitions of some parameters are quite different from our three-dimensional code. However, in this case, input parameters are selected in the same way if possible. The transverse distributions of initial beam radius are set as gaussian in the cylindrical coordinate, and the field distribution in the cavity is calculated with the assumption that the cavity is perfectly cylindrical. An emittance in Fig.2 is the normalized rms emittance, which is defined by $\epsilon_{x}=\langle\gamma\rangle\langle\beta\rangle \sqrt{\left\langle x^{2}\right\rangle\left\langle x^{\prime 2}\right\rangle-\left\langle x \cdot x^{\prime}\right\rangle^{2}}$ for our code, and $\epsilon_{r}=\frac{1}{2}\langle\gamma\rangle\langle\beta\rangle \sqrt{\left\langle r^{2}\right\rangle\left\langle r^{2}\right\rangle-\left\langle r \cdot r^{\prime}\right\rangle^{2}}$ for MAFIA.

Table 1: Calculation Parameters used in Fig.2

\begin{tabular}{|l|r|}
\hline Charge per bunch & $1.0[\mathrm{nC}]$ or none \\
Initial beam transverse profile & Gaussian \\
Initial beam radius on cathode & $1.5[\mathrm{~mm}](1 \sigma)$ \\
Longitudinal bunch profile & uniform \\
Bunch length & $10[\mathrm{ps}]$ \\
Initial emittance & $0[\pi \mathrm{mm} \cdot \mathrm{mrad}]$ \\
Maximum field in cavity & $150[\mathrm{MV} / \mathrm{m}]$ \\
Initial rf phase & $45[$ degree $]$ \\
MAFIA's mesh size & $0.1[\mathrm{~mm}]$ \\
\hline
\end{tabular}

Our code and MAFIA show good agreement when the space charge effects are ignored. However, our code shows

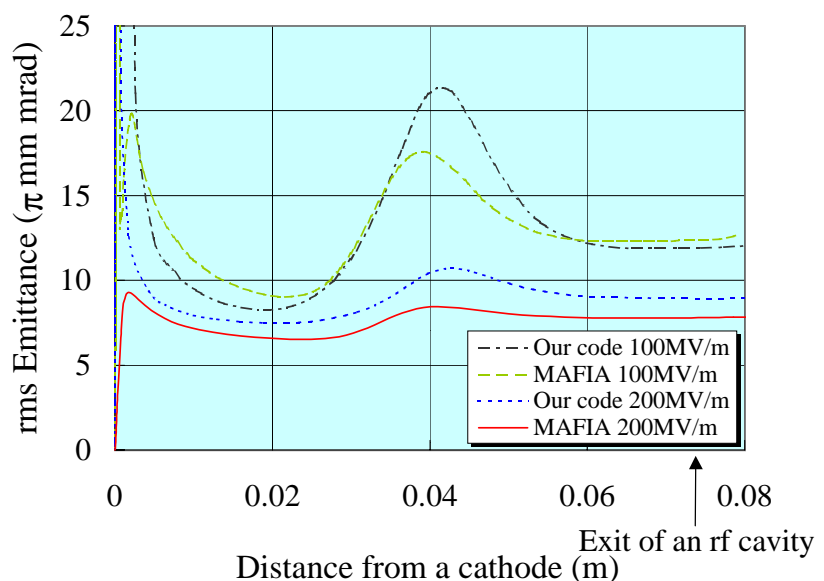

Figure 3: Comparison between our code and MAFIA. Charge is $1 \mathrm{nC}$ per bunch. Initial beam spot size is 0.25 $\mathrm{mm}(1 \sigma)$, which is almost comparable to MAFIA's mesh size. Maximum field in cavity varied as shown in the figure. Other parameters are the same as Table 1.

about $20 \%$ larger emittance than MAFIA in the calculation including charges of $1 \mathrm{nC}$ per bunch.

In the case of Fig.2, an initial beam radius is $1.5 \mathrm{~mm}$, because MAFIA's mesh size of $0.1 \mathrm{~mm}$ is selected. However, in the actual experiment, the laser beam spot size is expected to be more smaller. Fig.3 shows calaulation results for small size of $0.25 \mathrm{~mm}$ ( $1 \sigma)$, which corresponds to an actual spot size of the laser beam.

In Fig.2, magnitudes do not agree, but the distributions of the rms emittance show good agreement. However in Fig.3, even distributions do not agree when the fields in the cavity are low. This shows the limits of MAFIA's ability. In MAFIA, to set up more smaller mesh size or even threedimensional calculations are available, but more memories are needed. Moreover, if the fields in the cavity is low, the dependence on the space charge effects become large. Thus high calculation accuracy is required in these cases.

Our code also shows agreement with PERMELA, but it is useful when non-cylindrical fields or beams must be considered, or high accuracy is required.

\section{CALCULATIONS FOR THE TEST APPARATUS}

The calculation of the emittance varieties for the actual test apparatus were carried out using our code. In this section, we do not use the assumption of symmetrical cavity. The transverse distribution of the initial beam is set to be gaussian distribution in the rectangular coordinate. Fig. 4 shows the results that depend on the cavity field.

The emittance becomes lower as the field in the cavity increases. In a region over $200 \mathrm{MV} / \mathrm{m}$, it becomes almost constant. However, it is very difficult to increase the field of the cavity because of discharges or dark currents in actual experiment. 


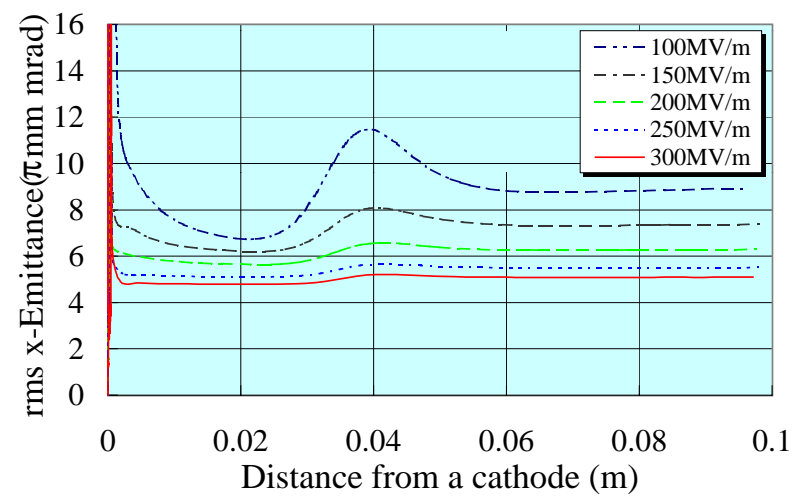

Figure 4: Emittance varieties depend on maximum cavity field changed from $100 \mathrm{MV} / \mathrm{m}$ to $300 \mathrm{MV} / \mathrm{m}$. Charge is 1 $\mathrm{nC}$ per bunch. Initial beam radius is $0.25 \mathrm{~mm}(1 \sigma)$. Other parameters are the same as Table 1.

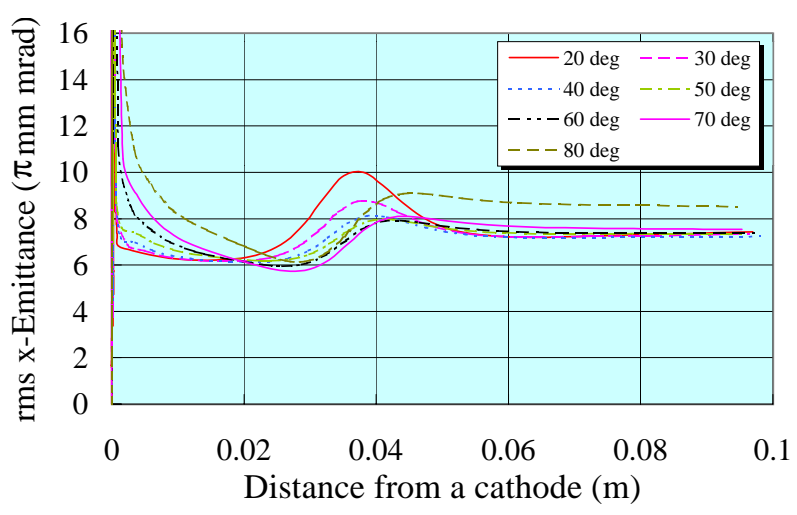

Figure 5: Emittance varieties depend on initial rf phase. Charge is $1 \mathrm{nC}$ per bunch. Initial beam radius is $0.25 \mathrm{~mm}$ $(1 \sigma)$. Maximum field in cavity is $150 \mathrm{MV} / \mathrm{m}$. Other parameters are the same as Table 1.

Figure 5 shows an initial phase dependence. As shown in Eq.4, if the initial phase is equal to zero, the field of the cavity becomes maximum when the laser is injected to the cathode surface. Therefore, the fields become lower as the initial phase increases. In a region from 20 degrees to 60 degrees, the emittances at the exit of the cavity are almost constant. This is because there is not so much difference in electron speed just after the cathode in this region, but in a region from 60 degrees to 80 degrees, the emittance becomes larger as the phase increases. This is because an electron speed just after the cathode becomes slower. In addition, a beam energy increases as the initial phase increases, and energy becomes almost constant of $3.5 \mathrm{MeV}$ with the cathode field of $150 \mathrm{MV} / \mathrm{m}$ in a region of over 60 degrees. Thus, the optimum initial phase is considered to be around 60 degrees.

Figure 6 shows the calculated emittances and beam sizes from the cathode to the Faraday-cup, which is located 1.5 $\mathrm{m}$ downstream of the cathode. This simulation includes the fields of the two solenoid coils. The first coil is positioned $0.174 \mathrm{~m}$ from the cathode, and the second coil is located at $0.374 \mathrm{~m}$. The $\mathrm{x}$ and $\mathrm{y}$ beam sizes are slightly different because of the asymmetry of the cavity.

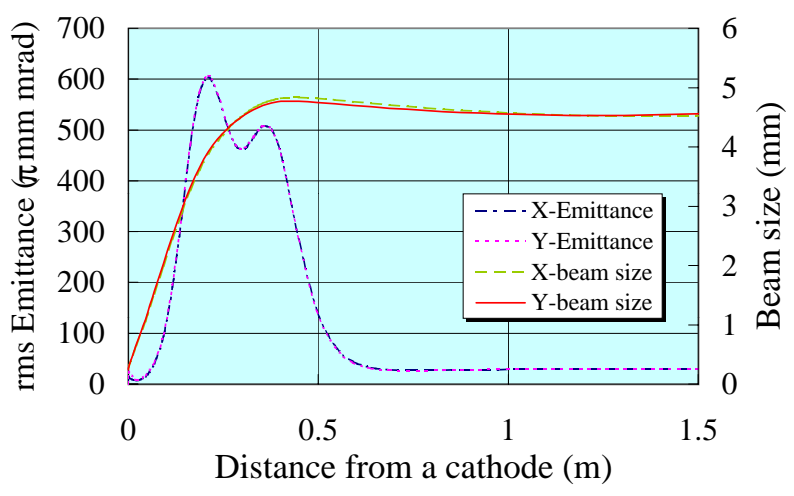

Figure 6: Calculated emittances and beam sizes from cathode to Faraday-cup. Parameters: $1 \mathrm{nC}$ charge, $0.25 \mathrm{~mm}$ $(1 \sigma)$ initial beam radius, $150 \mathrm{MV} / \mathrm{m}$ field in the cavity, 60 degrees initial rf phase, 400 number of particles. Field of first and second solenoid coil corresponds to 1500 and 800 Gausses, respectively.

The x-emittance and y-emittance change dramatically in the solenoid coils, because the $\mathrm{x}$ and $\mathrm{y}$ component of magnetic fields are coupled in solenoids, that is, the $\mathrm{x}$ component of the fields is the function of both $\mathrm{x}$ and $\mathrm{y}$, and even $\mathrm{z}$. Therefore, the emittance must not be constant. The emittance becomes smaller after the solenoid coils by choosing the optimum solenoid fields. In the experiment, we can measure the emittance only downstream of the solenoids, so when we estimate the emittance of the cavity, this calculation is very important.

The beam size fits our apparatus flange size of ICF70 in this condition. The calculated emittances are higher than expected. But main purpose of our test apparatus is to make comparisons in simulations.

\section{SUMMARY}

The particle tracking code including space charge effects for the rf gun test apparatus has been developed. Calculated emittances show good agreement with MAFIA and PERMELA. However, our code is suitable for precious or asymmetric calculations. In the near future, we are going to remove an assumption of uniform motion for electron in our code. Now, the experiment has just started. In actual measurements, new problems will be occurred, for instance, the transverse or longitudinal photon distributions of a laser pulse have a great influence on the emittance in our calculation. We are expected to observe these effects in practice.

\section{REFERENCES}

[1] T.Taniuchi et al., "Initial Results of RF Gun Experiment", this conf., New York, (1999). 